\title{
Distance Labeling in Hyperbolic Graphs
}

\author{
Cyril Gavoille* and Olivier Ly \\ LaBRI - Bordeaux University
}

\begin{abstract}
A graph $G$ is $\delta$-hyperbolic if for any four vertices $u, v, x, y$ of $G$ the two larger of the three distance sums $d_{G}(u, v)+d_{G}(x, y), d_{G}(u, x)+$ $d_{G}(v, y), d_{G}(u, y)+d_{G}(v, x)$ differ by at most $\delta$, and the smallest $\delta \geqslant 0$ for which $G$ is $\delta$-hyperbolic is called the hyperbolicity of $G$.

In this paper, we construct a distance labeling scheme for bounded hyperbolicity graphs, that is a vertex labeling such that the distance between any two vertices of $G$ can be estimated from their labels, without any other source of information. More precisely, our scheme assigns labels of $O\left(\log ^{2} n\right)$ bits for bounded hyperbolicity graphs with $n$ vertices such that distances can be approximated within an additive error of $O(\log n)$. The label length is optimal for every additive error up to $n^{\varepsilon}$. We also show a lower bound of $\Omega(\log \log n)$ on the approximation factor, namely every $s$-multiplicative approximate distance labeling scheme on bounded hyperbolicity graphs with polylogarithmic labels requires $s=\Omega(\log \log n)$.
\end{abstract}

Keywords. Distance queries, distance labeling scheme, hyperbolic graphs.

\section{Introduction}

It is well-known that a metric space $(V, d)$ embeds into a tree metric if and only if the 4-point condition holds, that is, for any 4 points $u, v, x, y$ of $V$ the two larger of the sums $d(u, v)+d(x, y), d(u, x)+d(v, y), d(u, y)+d(v, x)$ are equals [1]. More generally, if the two larger sums differ by at most $\delta$, then the metric space is said to be $\delta$-hyperbolic. Introduced by Gromov [21,20], $\delta$-hyperbolic spaces arise naturally in the area of geometric group theory. In a certain extend hyperbolicity measures the deviation from tree-likeness. And thus, it appears in a natural way as a generalization of the study of trees in metric graph theory [4, 5, 13], classification theory [8], phylogenetic analysis [29], and Gauber dynamics also known as Gibbs samplers [25].

A graph $G=(V, E)$ is $\delta$-hyperbolic if $\left(V, d_{G}\right)$ is a $\delta$-hyperbolic metric space, where $d_{G}$ is the shortest-path metric of $G$, associating to each pair of vertices the length of a shortest path connecting them. The hyperbolicity of $G$ is the smallest $\delta \geqslant 0$ for which $G$ is $\delta$-hyperbolic. The graphs considered in this paper are unweighted, simple, and connected.

\footnotetext{
* Supported by the project "PairAPair" of the ACI Masses de Données.
} 
0-hyperbolic graphs are precisely the block graphs [5, 12, 24], i.e., graphs in which every 2 -connected subgraph is a clique, and chordal graphs, i.e., the graphs containing no induced cycles of length larger than three, are 2hyperbolic [7]. It is not difficult to see from the definition that graphs of diameter $D$ are $(2\lfloor D / 2\rfloor)$-hyperbolic. 1-hyperbolic graphs have been partially characterized in [26], and recently a full characterization has been given in terms of a convexity condition and forbidden isometric subgraphs [3].

This paper deals with the problem of the distance computation and distributed abilities of $\delta$-hyperbolic graphs. Commonly, when we make a query concerning a set of nodes in a graph (adjacency, distance, connectivity, etc.), we need to make a global access to the structure. In our approach, the compromise is to store the maximum of information in a label associated with a vertex to have directly what we need with a local access. Motivation of localized datastructures in distributed computing is survey and widely discussed in [18].

We are especially interested in the distance labeling problem, introduced by Peleg in [30]. The problem consists in labeling the vertices of a graph in order to compute or estimate the distance between any two of its vertices $x$ and $y$ using only the information stored in the labels of $x$ and $y$, without any other source of information. The main parameters taken into account when designing a solution is the maximum label length (in bits) assigned by the labeling. More formally, an $(s, r)$-approximate distance labeling scheme on a given graph family $\mathfrak{F}$ is a pair $\langle L, f\rangle, L$ is called the labeling function and $f$ the distance decoder, such that, for every $G \in \mathfrak{F}$ and for all $x, y \in V(G): L(x, G) \in\{0,1\}^{*}$, and $d_{G}(x, y) \leqslant f(L(x, G), L(y, G)) \leqslant s \cdot d_{G}(x, y)+r$. If $s=1$ and $r=0$, then we shortly deal with a distance labeling scheme (or DLS). Also, an $(s, 0)$ approximate DLS is called $s$-multiplicative, and a $(1, r)$-approximate DLS is called $r$-additive.

Related works for distance labeling. The main results on the field are that general graphs support an (exact) distance labeling scheme with labels of $O(n)$ bits [19], and that trees [2,30], bounded tree-width graphs [19], distancehereditary graphs [16], bounded clique-width graphs [10], some non-positively curved plane graphs [9], all support distance labeling schemes with $O\left(\log ^{2} n\right)$ bit labels. Since 0-hyperbolic graphs are block graphs, which are distancehereditary, it follows that this class supports a $O\left(\log ^{2} n\right)$ bit label DLS.

The $O(n)$ bit upper bound is tight for general graphs (simply by counting the number of $n$-vertex graphs), and a lower bound of $\Omega\left(\log ^{2} n\right)$ bit on the label length is known for trees [19], implying that all the results mentioned above (including 0-hyperbolic graphs) are tight as well since all of them contains trees. Recently, [17,6] showed an optimal bound of $O(\log n)$ bits for interval graphs, 
permutation graphs, and their generalizations (circular-arc graphs and cicurlar permutation graphs).

Other results concern approximated distance labeling schemes. For arbitrary graphs, the best scheme in date is due to Thorup and Zwick [34]. They propose a $(2 k-1)$-multiplicative DLS, for each integral parameter $k \geqslant 1$, with labels of $O\left(n^{1 / k} \log ^{2} n\right)$ bits. Moreover, $\Omega\left(n^{1 / k}\right)$ bit labels are required in the worstcase for every $s$-multiplicative DLS with $s<2 k+1$. In fact, this result relies to a 1963 girth conjecture of Erdös [14] proved for $k=1,2,3$ and 5. However, for all the other values of $k$, the results of [27] implies that the $\Omega\left(n^{1 / k}\right)$ lower bound is true for $s<4 k / 3+2$.

In [15], it is proved that trees (and bounded tree-width graphs as well) enjoy a $(1+1 / \log n)$-multiplicative DLS with labels of $O(\log n \cdot \log \log n)$ bits, and this is tight in terms of label length and approximation. They also design some $O(1)$-additive DLS with $O\left(\log ^{2} n\right)$ bit labels for several families of graphs including: the graphs with bounded longest induced cycle, and, more generally, the graphs of bounded tree-length, i.e., that admit a Robertson-Seymour treedecomposition in bags of bounded diameter (see [11]). Interestingly, it is easy to show that every exact DLS for these families of graphs needs labels of $\Omega(n)$ bits in the worst-case (e.g., considering some chordal graphs, namely the split graphs [15]).

Recently, the graphs with doubling dimension $\alpha$ have been considered, i.e., the graphs for which, for every $r$, each ball of radius $2 r$ can be covered by at most $2^{\alpha}$ balls of radius $r$. It generalizes Euclidian metrics and bounded growth graphs, and includes many realistic networks. After several successive improvements [22,32,28], the best scheme in date, due to Slivkins [31], is a $(1+\varepsilon)$ multiplicative DLS with $O\left(\varepsilon^{-O(\alpha)} \log n \cdot \log \log n\right)$ bit labels. This is optimal for bounded $\alpha$ by combining the results of [28] and the lower bound of [15] for trees. Finally, in [33], it is shown that planar graphs enjoy a $(1+\varepsilon)$-multiplicative DLS with labels of $O\left(\varepsilon^{-1} \log ^{3} n\right)$ bits (see also [23]).

Our results. From the above list of results, it is clear that 0-hyperbolic graphs enjoy an (exact) DLS with $O\left(\log ^{2} n\right)$ bit labels, and that moreover every DLS for 2-hyperbolic graphs requires some labels of $\Omega(n)$ bits. Again, some chordal graphs, that are all 2-hyperbolic, require $\Omega(n)$ bit labels [15].

Our first contribution is a lower bound on an $s$-multiplicative DLS for bounded hyperbolicity graphs. We construct a family of bounded hyperbolic graphs for which, for every integer $k \geqslant 1$, every $s$-multiplicative DLS with $s<2 \log k+O(1)$ requires some labels of $\Omega(n / \log k)^{1 / k}$ bits. In particular, for $k=\Theta(\log n / \log \log n)$, it implies that any $s$-multiplicative DLS using labels of any poly-logarithmic length requires $s=\Omega(\log \log n)$. 
On the positive side, we construct for $\delta$-hyperbolic graphs an $\delta \log n$ additive DLS with labels of $O\left(\log ^{2} n\right)$ bits. The label length is optimal since every $r$-additive DLS for trees, and thus for $\delta$-hyperbolic graphs for every $\delta \geqslant 0$, requires $\Omega\left(\log ^{2}(n / r)\right)$ bit labels [19]. In the full version, we show that any poly$\log$ label DLS for bounded hyperbolic graphs requires $r=\Omega(\log n)$, proving the optimality of the approximation of our scheme.

Due to the lack of space, proofs appear in the full version.

\section{Pyramidal Construction}

Our lower bound combines several ingredients. First we show how to construct from any graph $G$ a graph $P$, called the pyramid of $G$, such that: 1) $G$ is a subgraph of $P$; 2) $P$ has bounded hyperbolicity (i.e., bounded by some constant independent of $G$ ); and 3) $d_{P}(x, y) \geqslant 2 \log d_{G}(x, y)-O(1)$ for all $x, y$ in $G$. In particular the girth of $P$ is at least the log of the girth of $G$.

Let $G$ be a graph. Let $D$ denote the diameter of $G$. Let us consider $\lceil\log D\rceil+$ 1 copies of $G$ denoted by $G_{0}, G_{1}, \ldots, G_{\lceil\log D\rceil}$. Let us consider a new graph constructed as follows: we start from the disjoint union of the $G_{i}$ 's. We add some new edge as follows: First, for any vertex $v$ of $G$, let us denote $v^{i}$ the copy of $v$ in $G_{i}$. For any $i=0, \ldots,\lceil\log D\rceil-1$, let us add an edge between $v^{i}$ and $v^{i+1}$. Such an edge shall be said to be vertical. Second, by induction, let us add a new edge between any two vertices of $G_{i}$, let say $v_{1}^{i}$ and $v_{2}^{i}$, if their copies in $G_{i-1}, v_{1}^{i-1}$ and $v_{2}^{i-1}$, are at distance 2 . Such an edge but also any orginal edge of some $G_{i}$ 's shall be said to be transversal. The graph that we obtain is denoted by $P(G)$, it is called the pyramid graph of $G, G_{0}$ is called the base of $P(G)$.

Lemma 1. There exists a constant $K$ such that for any $G$, the hyperbolicity of $P(G)$ is at most $K$.

Geodesics of $P(G)$. Here we consider the shape of geodesics of $P(G)$ in order to prove that $d_{P(G)}(x, y) \geqslant 2 \log d_{G}(x, y)-O(1)$ for all $x, y$ in $G$. The successive steps of this study are presented here along the following propositions. For any vertex $v$ of $P(G)$, let us call the height of $v$ the unique $i$ such that $v \in G_{i}$, it shall be denoted by $h(v)$. For any two vertices $v_{1}$ and $v_{2}$ of the same height $h$, we denote $d_{G_{h}}\left(v_{1}, v_{2}\right)$ the distance between $v_{1}$ and $v_{2}$ in the subgraph of $P(G)$ generated by the vertices of $G_{h}$. We denote by $D_{G_{h}}$ the maximum of $d_{G_{h}}$. If $p$ is a geodesic, i.e., a shortest path, then $\ell(p)$ denotes its length.

Proposition 1. Let $v_{1}$ and $v_{2}$ be two vertices of $G$. Then $d_{G_{h}}\left(v_{1}^{h}, v_{2}^{h}\right)=$ $\left\lceil d_{G}\left(v_{1}, v_{2}\right) / 2^{h}\right\rceil$. In particular $D_{G_{h}} \leqslant\left\lceil D / 2^{h}\right\rceil$. 
Proposition 2. Let p be a geodesic of $P(G)$ which only uses transversal edges. Then $\ell(p) \leqslant 5$.

Let us consider a path $p=v_{0} v_{1} \ldots v_{t}$ of length $t$. Let us consider the sequence of respective heights : $h_{0} h_{1} \ldots h_{t}$. We say that $p$ is increasing (resp. decreasing) if the sequence of heights is increasing (resp. decreasing).

Proposition 3. Let $p=v_{0} v_{1} \ldots v_{t}$ be a geodesic of $P(G)$. Let us assume that $h\left(v_{0}\right) \geqslant h\left(v_{t}\right)$. Then there exists a vertex $v_{i}$ of $p$ such that $v_{0} \ldots v_{i}$ is increasing and $v_{i} \ldots v_{t}$ is decreasing.

We consider a special kind of geodesic that we call straight geodesic. These are those having the following shape: first, it starts by using a sequence of vertical edges; second, it carries on by a sequence of transversal edges; and finally it uses a sequence of vertical edges.

Proposition 4. For any geodesic $p$, there exists a straight geodesic $p^{\prime}$ with same extremities. Moreover, $p$ is totally included into a 5-neighbourhood of $p^{\prime}$ and conversely.

Proposition 5. Let $x$ and $y$ be two vertices of $P(G)$. Let $p$ be such a straight geodesic between $x$ and $y$. Let us assume that $h(x) \leqslant h(y)$. Let $x^{\prime}$ be the copy of $x$ in $G_{h(y)}$. Let $h$ be the minimal of the lengths of the vertical parts of $p$ Then $\log \left(d_{G_{h(y)}}\left(x^{\prime}, y\right)\right)-3 \leqslant h \leqslant \log \left(d_{G_{h(y)}}\left(x^{\prime}, y\right)\right)-1$

The following proposition compares distances of $P(G)$ with those of the $G_{i}$ 's.

Proposition 6. Let $x$ and $y$ be two vertices of $P(G)$ with $h(x) \leqslant h(y)$. Let $x^{\prime}$ be the copy of $x$ in $G_{h(y)}$. Then $h(y)-h(x)+2 \log \left(d_{G_{h(y)}}\left(x^{\prime}, y\right)\right)-3 \leqslant$ $d_{P(G)}(x, y) \leqslant h(y)-h(x)+2 \log \left(d_{G_{h(y)}}\left(x^{\prime}, y\right)\right)+4$.

In particular, for all $x, y \in G, 2 \log \left(d_{G}(x, y)\right)-3 \leqslant d_{P(G)}(x, y) \leqslant$ $2 \log \left(d_{G}(x, y)\right)+4$.

Proposition 7. If $p$ and $p^{\prime}$ are two geodesics with same extremities, then $p$ is totally included into a 11-neighbourhood of $p^{\prime}$ and conversely.

Sketch of the Proof of Lemma 1 Let us be given with 3 vertices $x, y$ and $z$ of $P(G)$ (see Fig. 1). We consider 3 geodesics $p_{x y}, p_{y z}$ and $p_{x z}$ connecting respectively $x$ and $y, y$ and $z$, and $x$ and $z$. By the criterion of Rips (cf. [20]), it suffices to show that there exists a constant $K^{\prime}$, independent of $x, y$ and $z$, such that $p_{x z}$ is included into the $K^{\prime}$-neighbourhood of $p_{x y} \cup p_{y z}$. First, let us assume that $p_{x y}, p_{y z}$ and $p_{x z}$ are straight. We claim that in this case $p_{x z}$ is included into a 5-neighbourhood of $p_{x y} \cup p_{y z}$. Let us consider the notations indicated in Figure 1. Let us look at vertices of $p_{x z}$ case by case: 


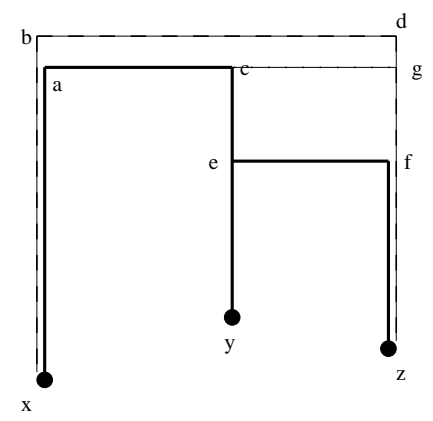

Fig. 1. Rips's Criterion.

- Vertices of $p_{x z}$ which are located between $x$ and $a$ belong also to $p_{x y}$.

- Without loss of generality, let us suppose that $p_{x y}$ is higher than $p_{y z}$. Vertices between $a$ and $b$ are at distance at most 3 from $p_{x y}$. Indeed, if $a$ is higher than $b$, it is true seeing that the segment $a b$ is totally included into $p_{x y}$. If $b$ is higher than $a$, one can verify the previous claim by applying Proposition 5 .

- By Proposition 2, vertices between $b$ and $d$ are at distance at most 5 from $b$, and therefore at most 8 from $a$.

- Vertices between $d$ and $g$ are within a distance at most 3 from $d$ and therefore at most 11 from $a$.

- Vertices between $g$ and $f$ are at most at distance 5 from the segment $e c$, because of the length of $e f$ which is at most 5 .

- Finally, vertices between $f$ and $z$ belong to $p_{y z}$.

We conclude that $p_{x z}$ is totally included into the 11-neighbourhood of $p_{x y} \cup p_{y z}$.

The general case where $p_{x y}, p_{y z}$ and $p_{x z}$ are not straight can be obtained from the above discussion by applying Proposition 4: we get that in general, $p_{x z}$ is included into the 21-neighbourhood of $p_{x y} \cup p_{y z}$

\section{Distance Labeling Lower Bound}

We consider the conjecture of Erdös according to which for any pair of integers $k \geqslant 1$ and $n \geqslant 1$, the maximal number of edges of a graph of girth $2 k+2$ with $n$ vertices is $\Omega\left(n^{1+1 / k}\right)$ (see [14]). It is true for $k=1,2,3,5$; it is also true if we consider graphs of girth $4 k / 3+3$ (see [27]). In the following, for any $k$ and $n$ we shall consider a graph $G_{n, k}$ of girth $4 k / 3+3$ with $n$ vertices and with maximal number of edges equal to $\Omega\left(n^{1+1 / k}\right)$.

We consider subgraphs defined by subsets of edges: given a graph $G$, a subset $E$ of edges of $G$ defines a subgraph $H$ whose vertices are the vertices of $G$ and whose edges are the elements of $E$. 
Proposition 8. Let us fix $k \geqslant 1$ and $n \geqslant 1$, and let us consider a subgraph $H$ of $G_{n, k}$. Let us consider $P(H)$ the pyramid graph of $H$, and a pair $(x, y)$ made of two vertices of the base of $P(H)$ which are connected by an edge in $G_{n, k}$. Then either $d_{P(H)}(x, y)=1$ or $d_{P(H)}(x, y) \geqslant 2 \log (4 k / 3+2)-3$.

Theorem 1. For $n \geqslant 1$ and $k \geqslant 1$, there exists a family $\mathfrak{F}_{n, k}$ of graphs of bounded hyperbolicity with $O(n \log k)$ vertices for which every $(s, r)$ approximated distance labeling scheme such that $s+r<2 \log (4 / 3 k+2)-3$ requires labels of $\Omega\left(n^{1 / k}\right)$ bits.

In particular, for $k=\Theta(\log n / \log \log n)$, every $s$-multiplicative DLS on $n$-vertex bounded hyperbolic graphs with poly-log label length requires $s=\Omega(\log \log n)$.

Proof. Let us consider the family $\mathfrak{F}_{n, k}$ of the pyramid graphs of the connected subgraphs of $G_{n, k}$. By maximality of the number of edges, it is not difficult to see that $G_{n, k}$ has diameter $O(k)$. We restrict ourself to connected subgraphs of diameter $O(k)$ by fixing some shortest path spanning tree in $G_{n, k}$. Observe that pyramid graphs that we obtain have $O(n \log k)$ vertices. By Lemma $1, \mathfrak{F}_{n, k}$ is of bounded hyperbolicity. Let us be given with an $(s, r)$-approximated distance labeling scheme $\langle L, f\rangle$ for $\mathfrak{F}_{n, k}$.

For each $H \in \mathfrak{F}_{n, k}$, let us denote by $S_{H}$ the word $L(1, H) \# L(2, H) \# \ldots \# L(n, H)$ obtained by concatenation of the labels of all the vertices of its base. We suppose that the vertex set of $G_{n, k}$ is $\{1,2, \ldots, n\}$. Besides, we use a special symbol \# as delimiter.

Let us assume that $\max _{H \in \mathfrak{F}_{n, k}, x \in V(H)}\{|L(x, H)|\}<c \cdot n^{1 / k}$ for some constant $c>0$. It follows that the number of words for $\mathfrak{F}_{n, k}$ is at most $2^{c \cdot n^{1+1 / k}}$. Because $\left|\mathfrak{F}_{n, k}\right|=2^{\left|E\left(G_{n, k}\right)-(n-1)\right|} \geqslant 2^{c^{\prime} \cdot n^{1+1 / k}}$ for some suitable constant $c^{\prime}>0$. This implies, for $c<c^{\prime}$ that there exists a pair $H_{1}$ and $H_{2}$ of distinct graphs of $\mathfrak{F}_{n, k}$ such that $L$ does not distinguish $H_{1}$ and $H_{2}$, i.e., $S_{H_{1}}=S_{H_{2}}$. Let us choose a pair of vertices $(x, y)$ of $G_{n, k}$ such that $(x, y)$ is an edge of the base of $H_{1}$ but not of the base of $H_{2}$. If such a pair does not exist, we exchange $H_{1}$ and $H_{2}$. If we cannot find such a pair, this means that $H_{1}=H_{2}$ which is a contradiction.

$S_{H_{1}}=S_{H_{2}}$ implies $L\left(x, H_{1}\right)=L\left(x, H_{2}\right)$ and $L\left(y, H_{1}\right)=L\left(y, H_{2}\right)$; and thus $f\left(L\left(x, H_{1}\right), L\left(y, H_{1}\right)\right)=f\left(L\left(x, H_{2}\right), L\left(y, H_{2}\right)\right)$.

Besides, by definition of $\langle L, f\rangle$, we have $d_{H_{1}}(x, y) \leqslant$ $f\left(L\left(x, H_{1}\right), L\left(y, H_{1}\right) \leqslant s \cdot d_{H_{1}}(x, y)+r\right.$ and $d_{H_{2}}(x, y) \leqslant$ $f\left(L\left(x, H_{2}\right), L\left(y, H_{2}\right) \leqslant s \cdot d_{H_{2}}(x, y)+r\right.$. 
All together we get $d_{H_{2}}(x, y) \leqslant s \cdot d_{H_{1}}(x, y)+r$. But $d_{H_{1}}(x, y)=1$ by assumption, and $d_{H_{2}}(x, y) \geqslant 2 \log (4 k / 3+2)-3$ by Proposition 8 . Finally we get $s+r \geqslant 2 \log (4 k / 3+2)-3$.

By contraposition, we have thus proved that for any $k$ and any $n, s+r<$ $2 \log (4 k / 3+2)-3$ implies that $\max _{H \in \mathfrak{F}_{n, k}, x \in V(H)}\{|L(x, H)|\} \geqslant c \cdot n^{1 / k}$.

\section{Tree Approximation and Distance Labeling}

This section is devoted to the proof of Theorem 2. It is based on the classical result about approximation of hyperbolic metric spaces by real trees (cf. e.g. [20, Thm. 12, p. 33]. We set up a combinatorial version of this result based on the same method of proof.

We use the characterization of hyperbolicity in terms of Gromov product. Let $G$ be a connected finite graph. Let $x, y$ and $w$ be vertices of $G$. One defines the Gromov product of $x$ and $y$ regarding $w$ to be $(x \mid y)_{w}=$ $\frac{1}{2}(|x-w|+|y-w|-|x-y|)$ where $|u-v|$ denotes $d_{G}(u, v)$. Let $G$ be a connected undirected finite graph. Then the hyperbolicity of $G$ is equal to $2 \max _{x, y, z, w \in G}\left\{\min \left\{(x \mid z)_{w},(z \mid y)_{w}\right\}-(x \mid y)_{w}\right\}$ (see [20]).

Proposition 9. Let $X$ be a finite 0-hyperbolic metric space with integral distances; let $D$ be the diameter of $X$. Then there exists a mapping $\sigma: X \rightarrow T$ where $T$ is a tree of at most $2(|X|-1) \cdot D$ nodes such that for any pair $(x, y)$ of elements of $X, d_{T}(x, y)=2 d_{X}(x, y)$.

Let $G$ be a connected undirected finite graph. Let us fix a vertex $w_{0}$ of $G$. In the following, $\left|x-w_{0}\right|$ shall be denoted by $|x|$ for any vertex $x$ of $G$, it shall be called the length of $x$ (regarding $w_{0}$ ). Following [20], let us define $(x \mid y)^{\prime}=\max \left\{\min _{2 \leqslant j \leqslant \ell}\left\{\left(x_{j-1} \mid x_{j}\right)_{w_{0}}\right\}\right.$ where $x_{1}, \ldots, x_{\ell}$ denotes any sequence of vertices. And from this, let $|x-y|^{\prime}=|x|+|y|-2(x \mid y)^{\prime}$.

Lemma 2. Let $\delta$ be the hyperbolicity of $G$. Then for any pair of vertices $x$ and $y$ of $G$, we have $|x-y|-\delta \log n \leqslant|x-y|^{\prime} \leqslant|x-y|$

Then we consider the equivalence relation defined by $x \equiv y$ if and only if $|x-y|^{\prime}=0$. And the metric space whose elements are $G / \equiv$ provided with the distance $d^{\prime}\left([x]_{\equiv},[y]_{\equiv}\right)=|x-y|^{\prime}$. We have the following property:

Lemma 3. $\left(G / \equiv, d^{\prime}\right)$ is a 0 -hyperbolic metric space.

Theorem 2. The family of $\delta$-hyperbolic graphs with $n$ vertices have a $\delta \log n$ additive distance labeling scheme with $O\left(\log ^{2} n\right)$ bit labels. 
Proof. Let us be given with $G$ a $\delta$-hyperbolic graph with $n$ vertices. We consider the mapping chain $G \stackrel{\pi}{\longrightarrow} G / \equiv \stackrel{\sigma}{\longrightarrow} T$ where $G \stackrel{\pi}{\longrightarrow} G / \equiv$ is defined as above and $G / \equiv \stackrel{\sigma}{\longrightarrow} T$ as in Proposition 9 (let us recall that $G / \equiv$ is 0-hyperbolic).

Since $T$ is a tree, there exists an exact distance labeling scheme $\left\langle L_{T}, f_{T}\right\rangle$ using labels of length $0\left(\log ^{2}|T|\right)$ (cf. [19]). By Proposition 9, $|T| \leqslant 2(n-1)^{2}$ because $|G| \equiv \mid \leqslant n$. So, labels used by $\left\langle L_{T}, f_{T}\right\rangle$ are of length $O\left(\log ^{2} n\right)$. Besides we have $|x-y|-\delta \log n \leqslant \frac{1}{2} d_{T}(\sigma \circ \pi(x), \sigma \circ \pi(y)) \leqslant|x-y|$.

Finally, let us define $L(x, G)=L_{T}(\sigma \circ \pi(x))$ and $f\left(\ell_{1}, \ell_{2}\right)=$ $\frac{1}{2} f_{T}\left(\ell_{1}, \ell_{2}\right)+\delta \log n$. Then $\langle L, f\rangle$ satisfies the conditions of the Theorem.

\section{References}

1. Richa Agarwala, Vineet Bafna, Martin Farach, Mike Paterson, and Mikkel Thorup. On the approximability of numerical taxonomy (fitting distances by tree metrics). In $7^{\text {th }}$ Symposium on Discrete Algorithms (SODA), pages 365-372. ACM-SIAM, January 1996.

2. Stephen Alstrup, Philip Bille, and Theis Rauhe. Labeling schemes for small distances in trees. In $14^{\text {th }}$ Symposium on Discrete Algorithms (SODA), pages 689-698. ACM-SIAM, January 2003.

3. Hans-Jürgen Bandelt and Victor D. Chepoi. 1-hyperbolic graphs. SIAM Journal on Discrete Mathematics, 16(2):323-334, 2003.

4. Hans-Jürgen Bandelt, A. Henkmann, and F. Nicolai. Powers of distance-hereditary graphs. Discrete Mathematics, 145:37-60, 1995.

5. Hans-Jürgen Bandelt and Henry Martyn Mulder. Distance-hereditary graphs. Journal of Combinatorial Theory, Series B, 41:182-208, 1986.

6. Fabrice Bazzaro and Cyril Gavoille. Localized and compact data-structure for comparability graphs. Research Report RR-1343-05, LaBRI, University of Bordeaux 1, 351, cours de la Libération, 33405 Talence Cedex, France, February 2005.

7. Gunnar Brinkmann, Jack H. Koolen, and Vincent Moulton. On the hyperbolicity of chordal graphs. Annals of Combinatorics, 5(1):61-65, 2001.

8. Peter Buneman. The recovery of trees from measures of dissimilarity. Mathematics in Archaeological and Historical Sciences, pages 387-395, 1971.

9. Victor D. Chepoi, Feodor F. Dragan, and Yann Vaxes. Distance and routing labeling schemes for non-positively curved plane graphs. Journal of Algorithms, 2005. To appear.

10. Bruno Courcelle and Rémi Vanicat. Query efficient implementation of graphs of bounded clique-width. Discrete Applied Mathematics, 131:129-150, 2003.

11. Yon Dourisboure and Cyril Gavoille. Tree-decomposition of graphs with small diameter bags. In J. Fila, editor, $2^{\text {nd }}$ European Conference on Combinatorics, Graph Theory and Applications (EUROCOMB), pages 100-104, September 2003.

12. Andreas W. M. Dress, Vincent Moulton, and Michael A. Steel. Trees, taxonomy, and strongly compatible multi-state characters. Advances in Applied Mathematics, 19:1-30, 1997.

13. Andreas W. M. Dress, Vincent Moulton, and Werner Terhalle. T-theory: an overview. European Journal of Combinatorics, 17:161-175, 1996.

14. Paul Erdös. Extremal problems in graph theory. In Publ. House Cszechoslovak Acad. Sci., Prague, pages 29-36, 1964. 
15. Cyril Gavoille, Michal Katz, Nir A. Katz, Christophe Paul, and David Peleg. Approximate distance labeling schemes. In F. Meyer auf der Heide, editor, $9^{\text {th }}$ Annual European Symposium on Algorithms (ESA), volume 2161 of Lecture Notes in Computer Science, pages 476-488. Springer, August 2001.

16. Cyril Gavoille and Christophe Paul. Distance labeling scheme and split decomposition. Discrete Mathematics, 273(1-3):115-130, 2003.

17. Cyril Gavoille and Christophe Paul. Optimal distance labeling schemes for interval and circular-arc graphs. In G. Di Battista and U. Zwick, editors, $11^{\text {th }}$ Annual European Symposium on Algorithms (ESA), volume 2832 of Lecture Notes in Computer Science, pages 254-265. Springer, September 2003.

18. Cyril Gavoille and David Peleg. Compact and localized distributed data structures. Journal of Distributed Computing, 16:111-120, May 2003. PODC 20-Year Special Issue.

19. Cyril Gavoille, David Peleg, Stéphane Pérennès, and Ran Raz. Distance labeling in graphs. Journal of Algorithms, 53(1):85-112, 2004.

20. Etienne Ghys and Pierre de La Harpe. Sur les Groupes Hyperboliques d'après Mikhael Gromov. Birkhäuser, 1990.

21. Mikhael Gromov. Hyperbolic groups. Essays in Group Theory, Mathematical Sciences Research Institute Publications, 8:75-263, 1987.

22. Anupam Gupta, Robert Krauthgamer, and James R. Lee. Bounded geometries, fractals, and low-distortion embeddings. In $44^{\text {th }}$ Annual IEEE Symposium on Foundations of Computer Science (FOCS), pages 534-543. IEEE Computer Society Press, October 2003.

23. Anupam Gupta, Amit Kumar, and Rajeev Rastogi. Traveling with a pez dispenser (or, routing issues in mpls). SIAM Journal on Computing, 34(2):453-474, 2005.

24. Edward Howorka. On metric properties of certain clique graphs. Journal of Combinatorial Theory, Series B, 27:67-74, 1979.

25. Haim Kaplan and Tova Milo. Parent and ancestor queries using a compact index. In $20^{\text {th }}$ ACM Symposium on Principles of Database Systems (PODS). ACM-SIAM, May 2001.

26. Jack H. Koolen and Vincent Moulton. Hyperbolic bridged graphs. European Journal of Combinatorics, 23(6):683-699, 2002.

27. Felix Lazebnik, Vasiliy A. Ustimenko, and Andrew J. Woldar. A new series of dense graphs of high girth. Bulletin of the American Mathematical Society (New Series), 32(1):73-79, January 1995.

28. Manor Mendel and Sariel Har-Peled. Fast construction of nets in low dimensional metrics, and their applications. In $21^{\text {st }}$ Annual ACM Symposium on Computational Geometry (SoCG), pages 150-158, 2005.

29. Vincent Moulton and Michael A. Steel. Retractions of finite distance functions onto tree metrics. Discrete Applied Mathematics, 91:215-233, 1999.

30. David Peleg. Proximity-preserving labeling schemes. Journal of Graph Theory, 33:167-176, 2000.

31. Aleksandrs Slivkins. Distance estimation and object location via rings of neighbors. In $24^{\text {th }}$ Annual ACM Symposium on Principles of Distributed Computing (PODC), pages 41-50. ACM Press, 2005.

32. Kunal Talwar. Bypassing the embedding: Algorithms for low dimensional metrics. In $36^{\text {th }}$ Annual ACM Symposium on Theory of Computing (STOC), pages 281-290, June 2004.

33. Mikkel Thorup. Compact oracles for reachability and approximate distances in planar digraphs. Journal of the ACM, 51(6):993-1024, November 2004.

34. Mikkel Thorup and Uri Zwick. Approximate distance oracles. Journal of the ACM, 52(1):124, January 2005. 